\title{
Systematic Correct Construction of Self-stabilizing Systems: A Case Study ${ }^{\star}$
}

\author{
Ananda Basu ${ }^{2}$, Borzoo Bonakdarpour ${ }^{1}$, Marius Bozga ${ }^{2}$, and Joseph Sifakis ${ }^{2}$ \\ 1 Department of Electrical and Computer Engineering \\ University of Waterloo \\ 200 University Avenue West \\ Waterloo, Ontario, Canada, N2L 3G1 \\ 2 VERIMAG \\ 2 Avenue de Vignate \\ 38610, Gières, France
}

\begin{abstract}
Design and implementation of distributed algorithms often involve many subtleties due to their complex structure, non-determinism, and low atomicity as well as occurrence of unanticipated physical events such as faults. Thus, constructing correct distributed systems has always been a challenge and often subject to serious errors. We present a methodology for component-based modeling, verification, and performance evaluation of self-stabilizing systems based on the BIP framework. In BIP, a system is modeled as the composition of a set of atomic components by using two types of operators: interactions describing synchronization constraints between components, and priorities to specify scheduling constraints. The methodology involves three steps illustrated using the distributed reset algorithm due to Arora and Gouda. First, a high-level model of the algorithm is built in BIP from the set of its processes by using powerful primitives for multi-party interactions and scheduling. Then, we use this model for verification of properties of a self-stabilizing algorithm including closure, deadlock-freedom, and finite reachability of the set of legitimate states. Finally, a distributed model which is observationally equivalent to the high-level model is generated. This model is used for performance analysis taking into account the degree of parallelism and convergence times for failure-free behavior as well as in the presence of faults.
\end{abstract}

Keywords: Component-based modeling, Verification, Selfstabilization, Distributed algorithms, Reset algorithms.

\section{Introduction}

Distributed systems are constructed from a set of relatively independent components that form a unified, but geographically and functionally diverse entity.

\footnotetext{
* This is an extended version of the paper presented at SSS'10. This work is sponsored by the COMBEST European project. For all correspondence, please contact Borzoo Bonakdarpour at borzoo@ecemail.uwaterloo.ca.
} 
They remain difficult to design, build, and maintain, because of their inherently concurrent, non-deterministic, and non-atomic structure as well as the occurrence of unanticipated physical events such as faults.

We currently lack disciplined methods for rigorous design and correct implementation of distributed systems. These systems are still being constructed in an ad-hoc fashion in practice, mainly for two reasons: (1) formal methods are not easy to use by engineers; and (2) there is a wide gap between modeling formalisms and automated verification tools on one side, and practical development and deployment tools on the other side. In fact, it is not clear how existing results can be consistently integrated in design and implementation methodologies. Formalisms such as process algebras [1], I/O automata [17,23], and UNITY [10] have been used for modeling and reasoning about the correctness of distributed systems. These methods are either too formal to be used by engineers, or, they require the designer to specify low-level elements of a distributed system such as channels and schedulers [23]. Numerous techniques and algorithms have also been introduced for adding reliability and fault-tolerance to distributed systems. Moreover, an interest has recently emerged in verification of distributed algorithms. While these approaches play an important role in formalizing and achieving correctness of distributed algorithms, we believe that a more practical systematic approach for modeling, verification, and as importantly deployment of distributed systems is still required.

In this paper, we apply a methodology which consistently integrates modeling, verification, and deployment techniques, based on the BIP (Behavior, Interaction, Priority) framework [3,4]. BIP is based on a semantic model encompassing composition of heterogeneous components. In contrast to all other formalisms using a single type interaction (e.g., rendezvous, asynchronous message passing), BIP uses two families of composition operators for expressing coordination between components: interactions and priorities. Interactions are expressed by combining two protocols: rendezvous and broadcast, which makes BIP more expressive than any formalism based on a single type of interaction [6]. Supporting tools of BIP's theory include techniques for model verification [20] as well as for generating from a high-level model an observationally equivalent multi-threaded or distributed implementation $[3,7,8]$.

To illustrate our methodology, we focus on self-stabilizing systems. Pioneered by Dijkstra [11], a self-stabilizing distributed algorithm guarantees that starting from an arbitrary state, it converges to a legitimate state (from where it satisfies its specification) and remains thereafter. As Dijkstra points out in a belated proof of correctness of his token ring algorithm [12], designing and deploying correct self-stabilizing algorithms is not a trivial task at all, although it initially seems straightforward. We describe our methodology to overcome these difficulties using the distributed reset self-stabilizing algorithm [2]. We demonstrate how refinement of a simple algorithm to a less high-level model involves many subtleties that may dramatically affect the correctness of the refined model. We also show how BIP facilitates rigorous modeling, verification, and performance analysis of the distributed reset algorithm. Our methodology involves three steps: 
- The starting point is a high-level BIP model of a distributed system obtained as the composition of a set of components. This model represents a system with a global state and atomic transitions. Interactions may lead the system from one global state to another. Modeling a distributed system in such a high-level model confers numerous advantages such as modularity by using abstract behavioral components and faithfulness as coordination is directly expressed by using abstract multi-party interactions instead of lowlevel primitives. We also show how different functions of a self-stabilizing system (e.g., normal as well as recovery) can be elegantly modeled in BIP in an incremental manner.

- We use this compact high-level model for verification of safety and liveness properties that any self-stabilizing algorithm must satisfy. These properties include closure, deadlock-freedom, and finite reachability of the set of legitimate states. We verify these properties on our BIP model for distributed reset by using model checking techniques.

- Finally, a multi-threaded or distributed executable $\mathrm{C}++$ code is automatically generated from the high-level model for simulations and experiments $[3,7,8]$. This $\mathrm{C}++$ code faithfully represents an actual multi-threaded or distributed implementation of the high-level model. It is obtained by applying two transformations preserving observational equivalence $[3,7,8]:(1)$ multi-party interactions are substituted by protocols based on asynchronous message passing; (2) the state of a component is undefined (due to concurrency) when it performs some internal computation. In this paper, we use a multi-threaded implementation in order to conduct guided simulations for estimating performance of distributed reset. This includes analysis taking into account the degree of parallelism and convergence times for failure-free behavior as well as in the presence of faults.

Organization of the paper. In Section 2, we review the distributed reset algorithm and basic concepts of the BIP framework. In Section 3, we formally model distributed reset in BIP. Section 4 is dedicated to verification of distributed reset. We describe our experiments and analyze the performance of distributed reset in Section 5. Finally, we conclude in Section 6.

\section{Background}

\subsection{Distributed Reset}

Intuitively, distributed reset [2] augments functionality of a distributed system with a subsystem where each process can initiate a global reset to a predefined global state. Each process is associated with a set of adjacent processes with which it can communicate. At any time instant, an alive process may crash which results in change of the list of adjacent processes. The reset subsystem consists of the following three layers (see Figure 1-a):

- In the tree layer, adjacent processes communicate in order to construct and maintain a rooted spanning tree throughout the alive processes. Thus, any 


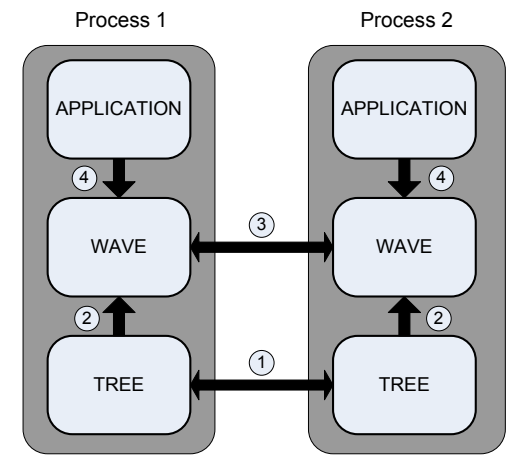

(a) Two adjacent processes in distributed reset.

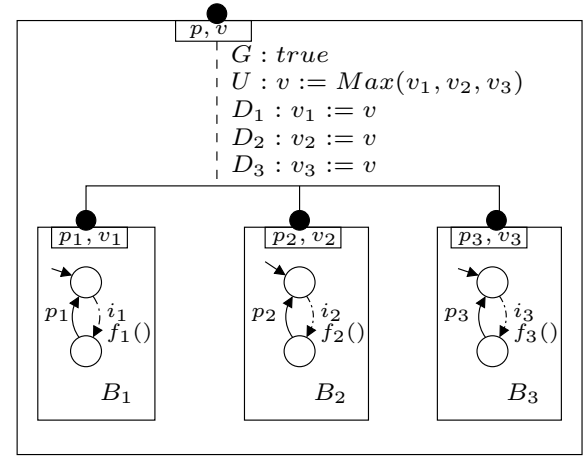

(b) A simple BIP model.

Fig. 1. Preliminary concepts.

changes in the adjacency relationship of processes eventually result in corresponding changes in the structure of the spanning tree. The tree layer is self-stabilizing in that starting from any arbitrary topology and initial structure, construction of a rooted spanning tree within a finite number of steps is guaranteed. Thus, faults such as process failures and local variable corruptions do not result in permanent destruction of the spanning tree. Communication among these processes establish Channel 1 in Figure 1-a.

- The application layer may locally choose to initiate a global reset. In this case, the corresponding local component sends a request to the local wave layer described next (see Channel 4 in Figure 1-a).

- The wave layer may receive a reset request from the application layer in order to start a global reset. In this case, the local wave component of a process forwards the request to its parent in the current spanning tree until the request reaches the root. Once the root receives a reset request, it initiates a diffusing computation as follows. First, the root resets its own state and then initiates a reset wave. The reset wave travels towards the leaves of the spanning tree and causes the wave component of each encountered process to reset its state. When the reset wave reaches a leaf process, it bounces as a completion wave that travels towards the root process. A process propagates the completion wave to its parent if all its offsprings are complete (see Channel 3 in Figure 1-a). When the completion wave reaches the root, the global reset is complete. Each wave component maintains a session number in order to ensure that concurrent resets do not interfere. The wave layer is also self-stabilizing in the sense that starting from any arbitrary configuration of the wave components, the algorithm guarantees an eventual global reset within a finite number of steps. The wave layer always assumes the existence 
of a sound rooted spanning tree. Thus, the only piece of information that a tree component shares with the corresponding local wave component is the identity of the parent process in the spanning tree (see Channel 2 in Figure $1-\mathrm{a})$.

\subsection{The BIP Framework}

In the BIP language $[4,5,22]$, an architecture is characterized as a hierarchically structured set of components obtained by composition from a set of atomic components. Composition is parameterized by sets of interactions between the composed components. The BIP toolset has a compilation chain allowing the generation of different types of $\mathrm{C}++$ code (e.g., monolithic, real-time, multithreaded, distributed, etc) from BIP models. The generated code is modular and can be executed on a dedicated middleware consisting of one or more Engines that orchestrate the computation of atomic components by executing their interactions. Hierarchical description allows incremental reasoning and progressive design of complex systems. Priorities among interactions allow specifying scheduling policies in BIP.

A BIP component is characterized by its interface and its behavior. An interface consists of a set of external ports used to specify interactions. Each port $p$ is associated with a set $v_{p}$ of variables which are visible when an interaction involving $p$ is executed. It is assumed that the ports and associated variables of atomic components are disjoint. The behavior of atomic components is described as a finite state automaton extended with data and functions given in $\mathrm{C}++$. A transition of the automaton is labeled by (1) a port $p$ through which an interaction is sought, (2) a function $f$ describing a local computation, and (3) a guard $g$ on local data. For a given control state, a transition can be executed if its guard $g$ is true and an interaction involving $p$ is possible (we precisely define the notion of interactions later in this section). Execution of transitions is atomic: it is initiated by the interaction and followed by the execution of $f$. A component may have internal ports as well. Transitions labeled by internal ports are executed independently and do not require initiation of an interaction.

Graphical notation. An atomic component (i.e., its behavior and interface) is placed in a box (see Figure 1-b). Each external port and its corresponding variables are placed in a rectangle inside its containing component. Behavior of a component is described by the classic notation of an automaton. We use a solid (respectively, dotted) arrow to denote a transition labeled by an external (respectively, internal) port.

Composition consists of applying a set of connectors to a set of components. A connector is defined by:

1. its support set of ports $\left\{p_{1}, \ldots, p_{n}\right\}$ of the composed components;

2. optionally an exported port $p$ by the connector and the associated variables;

3 . its set of interactions, that are, subsets of the set $\left\{p_{1}, \ldots, p_{n}\right\}$. Each interaction $\alpha=\left\{p_{i_{1}} \ldots p_{i_{k}}\right\}$ is annotated by 
(a) a guard $G$, Boolean expression involving variables associated with the ports $p_{i_{j}}$ involved in the interaction $\alpha$;

(b) an upstream transfer function $U$ specifying flow of data from variables associated with the support set of ports towards the associated variables of the exported port;

(c) and downstream transfer functions $D_{i_{1}}, \ldots, D_{i_{k}}$ specifying flow of data from the variables associated with the exported port towards variables associated with the support set of ports.

When it is clear from the context, we simply denote a connector by only its support set of ports (i.e., $\left.\left\langle p_{1} \ldots p_{n}\right\rangle\right)$. The set of interactions associated with a connector is defined using a typing mechanism of ports in its support set of ports. We distinguish two types of ports: synchron and trigger. Any set of support ports that is either maximal or it contains a trigger denotes a valid interaction. Intuitively, a synchron is a passive port, and needs synchronization with other ports. In other words, such a port cannot initiate an interaction without synchronizing with other ports. However, a special case (such as the one in Figure 1-b) is a connector that only involves synchrons. Such a connector denotes a rendezvous and requires all ports to participate. On the other hand, a trigger is an active port, and can initiate an interaction without synchronizing with other ports. The global behavior resulting from the application of a connector to a set of components is defined as follows. An interaction $\alpha=\left\{p_{i_{1}} \ldots p_{i_{k}}\right\}$ of the connector is enabled only if for each one of its ports $p_{i_{j}}$, there exists an enabled transition in some component labeled by $p_{i_{j}}$. Execution of the interaction involves two steps:

1. a temporary variable $v$ is assigned the value $U\left(v_{p_{i_{1}}}, \ldots, v_{p_{i_{k}}}\right)$;

2. the variables $v_{i_{j}}$ associated with the ports $p_{i_{j}}$ are assigned values $D_{i_{j}}(v)$.

The execution of an interaction is followed by the execution of the local computations of the synchronized transitions. A composite component is recursively obtained from a set of atomic or sub-components by successive (i.e., acyclic) application of connectors. The support set of any connector contains ports exported either by sub-components or other existing connectors.

Graphical notation. A connector is represented as a solid line connecting all ports in its support set. The exported port by a connector is placed over the connector. A solid circle attached to an external port denotes a synchron and a triangle denotes a trigger (see Figure 2-b). A composite component is also respresented as a box containting its sub-components and their respective connector hierarchy.

In Figure 1-b, we provide a simple composite component. It is composed of three atomic components $B_{1}, B_{2}$, and $B_{3}$. Each atomic component $B_{k}$ holds an integer variable $v_{k}$, exported through an external port $p_{k}$. Additionally, the component has an internal port $i_{k}$ which triggers the execution of an internal computation defined by the function $f_{k}$. The ternary connector defines the interaction $\left\{p_{1}, p_{2}, p_{3}\right\}$ which is a rendezvous among external ports $p_{1}, p_{2}$, and $p_{3}$. As 
a result of this interaction, following the definition of upstream an downstream transfer functions, each component receives the maximum of the exported values. Notice that the exported port of the connector belongs to the interface of the composite component, that is, it can be used for further interactions.

\section{Modeling Distributed Reset in BIP}

We model distributed reset according to the BIP system construction methodology: (1) designing the behavior of each atomic component (i.e., an automaton extended by variables and ports), (2) applying synchronization mechanisms for ensuring coordination of distributed components through interactions, and (3) specifying scheduling constraints by using priorities. We apply this methodology to model the wave layer and the tree layer in a modular manner in Subsections 3.1 and 3.2, respectively. Then, we add cross-layer connectors in Subsection 3.3. We also systematically model normal, recovery, and faulty behaviors of distributed reset using independent interactions. From the wave and tree components designed in this section, one can incrementally build a distributed system equipped with the distributed reset functionality according to a topology of interest.

\subsection{The Wave Layer}

The wave layer of distributed reset assumes that a sound rooted spanning tree exists throughout the distributed system. Thus, the wave layer is only concerned with achieving a self-stabilizing diffusing computation to accomplish a distributed reset. Each process in the distributed system contains a wave atomic component.

Normal Operation We start with modeling the normal operation of the wave layer, where each component works correctly in the absence of faults.

\section{Interface and Behavior}

- (Exported Ports) A wave component has the following four ports: (1) $p$ Request for propagating a reset request from a child to its parent, (2) pReset for enforcing a child to reset its state by the parent, (3) pComplete for informing a node that its subtree has completed diffusing computation, and (4) $p P c$ for identifying adjacent processes that are neither a child nor a parent. As can be seen in Figure 2-a, each port is associated with a subset of variables of the component.

- (Variables) Each component maintains the following variables: (1) an integer index to represent the unique index of the component, (2) an integer $f$ to keep the index of the parent process in the spanning tree, and (3) an integer $s n$ for the session number of the current ongoing reset. 


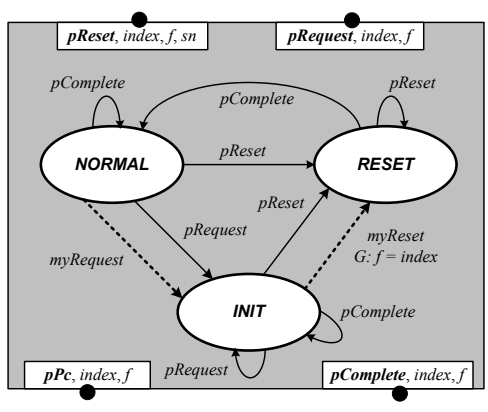

(a) Behavior and interface

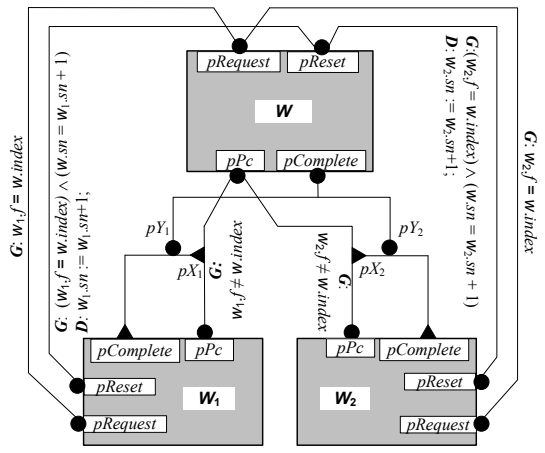

(b) Interactions

Fig. 2. Normal operation of the wave layer.

- (Automaton) A wave component has three control states: NORMAL, INIT, and RESET (see Figure 2-a). Initially, all components are in the NORMAL control state. A wave component may move to INIT by either enabling the myRequest internal port (e.g., from the application layer of the same process) or when a reset request is received via the $p$ Request port. This move occurs during the request wave. Next, the component moves from INIT to RESET and resets its state when the port pReset is enabled during the reset wave. A component may also move from INIT to RESET on port pReset, if it was not involved in the request wave. Finally, a wave component moves back to NORMAL on port $p$ Complete, when its subtree has completed the completion wave. A completed wave component is either in NORMAL control state or in INIT if another reset has already been initiated in its subtree. The $p$ Complete selfloop at this control state is added for this reason.

\section{Interactions}

Interactions among wave components are specified in terms of a set of connectors between them. Notice that each process is associated with a set of adjacent processes according to a topology. In order to make our design as flexible as possible, the static design of connectors should provide the potential of communication between any two adjacent processes depending upon the topology. Nonetheless, the actual communication in the wave layer should occur only between processes that are allowed to do so by the parent-child relationship determined by the tree layer. This is similar to designing a circuit of electronic components with a set of switches, where depending upon the state of switches only a subset of wires between components work. Let $w$ be a wave component whose adjacent neighbors are $w_{1} \cdots w_{n}$. We categorize the interactions based on the three waves of the wave layer. These connectors construct Channel 3 of Figure 1-a: 
- (Request Wave) The first set of connectors is $\left\{\left\langle(w . p R e q u e s t)\left(w_{i} \cdot p\right.\right.\right.$ Request $\left.)\right\rangle \mid$ $1 \leq i \leq n\}$. These connectors allow the component $w$ at NORMAL to synchronize with a component $w_{i}$, that is already in control state INIT: $w_{i}$ synchronizes with $w$ by taking the pRequest self-loop at control state INIT. Figure 2 -b presents an example, where $w$ has two adjacent processes $w_{1}$ and $w_{2}$. The connectors between pRequest ports are associated with a guard to ensure correct parent-child relationship and bottom-up flow of requests (e.g., $w$. index $\left.=w_{1} . f\right)$. Hence, if two processes are adjacent due to the topology, but not in any parent-child relationship, they do not interact to send or receive reset requests. This guard is present in almost all of the connectors in the wave layer. Symmetric conditions in adjacent processes (e.g., $w_{1}$ is parent of $w$ ) are omitted from the figure for simplicity. Recall that since BIP allows us to associate ports with variables, evaluation of the above guard does not require explicit use of shared memory.

- (Reset wave) The second set of connectors is $\left\{\left\langle(w . p R e s e t)\left(w_{i} \cdot p\right.\right.\right.$ Reset $\left.)\right\rangle \mid 1 \leq$ $i \leq n\}$. Once the root (of the spanning tree) wave component moves to INIT, it goes to RESET without synchronizing on port pReset. This is managed through specifying an internal transition from INIT to RESET with guard $(w . f=w . i n d e x)$. Once a process is in RESET, its children can go to RESET from either NORMAL or INIT by synchronizing on port pReset. In other words, a child whose parent is in RESET can reset its state regardless of its past desire to initiate a global reset. A parent synchronizes with its resetting children through the $p$ Reset self-loop at control state RESET. Similar to the connector between pRequest ports, we ensure that only a parent can propagate the reset wave to its children by specifying a guard on the connector between pReset ports. This guard also ensures that the session number of a child is one less than the session number of its parent. Finally, when the reset connector gets enabled, it increments $s n$ of the child component to mark the session number of the current reset wave.

- (Completion wave) A process declares completion only if all its children are complete (which essentially means its entire subtree is complete). The completion mechanism inherently requires a multi-party rendezvous. However, our design should be flexible in that it allows bypassing adjacent processes that are neither a parent nor a child. To this end, we construct a hierarchical connector as follows. First, we include a connector between $p P c$ ports of $w$ and $w_{i}$, where $1 \leq i \leq n$, which gets enabled when $w$ and $w_{i}$ are not in a parent-child relationship. This connector exports the trigger port $p X_{i}$, which gets enabled when the completion of $w_{i}$ is irrelevant to $w$. Now, the pair of $p X_{i}$ and $w_{i}$.p Complete constructs another connector, which exports the port $p Y_{i}$. This port is present in the rendezvous that covers all $w_{i}$ components. The full interaction can be characterized by the following rendezvous: $\left\langle(w . p\right.$ Complete $\left.) p Y_{1} p Y_{2} \cdots p Y_{n}\right\rangle$, where $p Y_{i}=\left\langle\left(p X_{i}\right)+\left(w_{i} \cdot p\right.\right.$ Complete $\left.)\right\rangle$ and $p X_{i}=\left\langle(w . p P c)\left(w_{i} \cdot p P c\right)\right\rangle$. The '+' operator denotes a choice between two enabled ports. 


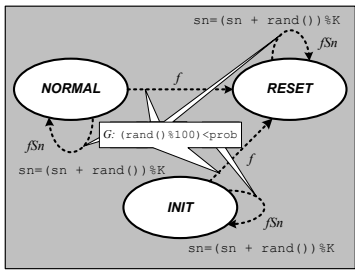

(a) Faulty behavior

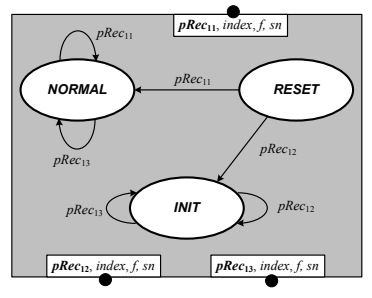

(b) Recovery type 1

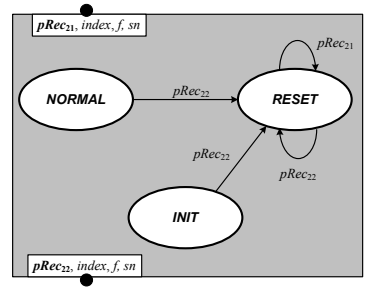

(c) Recovery type 2

Fig. 3. Self-stabilization of the wave layer.

Notice that starting from an initial state and operating normally, the global state of the set of all components in the wave layer arranged on a rooted spanning tree should remain in the following set of legitimate states, for any two wave components $w_{1}$ and $w_{2}$ :

$$
\begin{aligned}
& \mathcal{S}_{w} \equiv \forall w_{1}, w_{2}::\left(\left(w_{1} . f=w_{2} . \text { index } \wedge \neg w_{2} . R E S E T\right) \Rightarrow\right. \\
& \left.\left(\neg w_{1} \cdot R E S E T \wedge w_{1} . s n=w_{2} . s n\right)\right) \wedge \\
& \left(\left(w_{1} . f=w_{2} . \text { index } \wedge \quad w_{2} . R E S E T\right) \Rightarrow\right. \\
& \left.\left(\left(\neg w_{1} . R E S E T \wedge w_{2} . s n=w_{1} . s n+1\right) \vee w_{2} . s n=w_{1} . s n\right)\right) .
\end{aligned}
$$

Faulty Behavior In distributed reset, faults can lead a process to reach any arbitrary state in $\neg \mathcal{S}_{w}$ (See Figure 3-a). The transitions labeled by internal port $f$ cause a process to go to RESET from either INIT or NORMAL without synchronizing with its parent. Faults labeled by $f S n$ are self-loops that corrupt the session number of a process by executing the $\mathrm{C}++$ instruction $\mathrm{sn}=(\mathrm{sn}+\operatorname{rand}())$ $\% \mathrm{~K}$, where $\mathrm{K}$ is the maximum number of processes. To make the occurrence of faults a random event, we associate the guard of fault transitions with a probability prob. Notice that the union of transitions in Figures 2-a and 3-a may lead a wave component to reach any arbitrary state. Finally, fault transitions are labeled by internal ports making their occurrence independent of synchronization constraints.

\section{Self-stabilization}

Interface and Behavior. We model self-stabilization of the wave layer based on violation of either conjuncts of $\mathcal{S}_{w}$. Essentially, the recovery mechanism should ensure that starting from any state in $\neg \mathcal{S}_{w}$, the entire distributed system can reach a state in $\mathcal{S}_{w}$ within a finite number of steps. For the first conjunct (see Figure 3-b), first, we consider the case where a parent process is not in RESET, but one of its children is. To resolve this case, it suffices for the child to (1) move to 
the control state where its parent is (i.e., either NORMAL through synchronization on port $p R e c_{11}$ or INIT through port $p \operatorname{Rec}_{12}$ ), and (2) copy the session number from the parent to ensure consistency. Then, to resolve the case where a parent and its child are in the same control state but their session numbers differ, the processes synchronize on port $p \operatorname{Rec}_{13}$ and the child copies the parent's session number.

For the second conjunct (see Figure 3-c), if a process and one of its children are in RESET, but their session numbers differ, then they synchronize on port $p R e c_{21}$ and the child copies the session number. Finally, if a process is in RESET, but one of its children is not in RESET and the child's session number is not one less than its parent's, then they synchronize on port $p R e c_{22}$ and the child copies the session number.

Interactions. Recovery connectors define interactions on corresponding ports between adjacent components. Thus, the set of connectors is $\left\{\left\langle\left(w . p \operatorname{Rec}_{j k}\right)\left(w_{i} \cdot p \operatorname{Rec}_{j k}\right)\right\rangle \mid(i=1 . . n) \wedge(j=1 . .2) \wedge(k=1 . .3)\right\}$, where $w_{i}$ is adjacent to $w$. Similar to the normal operation, we associate guards with recovery connectors to ensure the correct parent-child relationship among the adjacent processes. Moreover, we incorporate data transfer as explained in Subsection 2.2 in interactions for copying session numbers.

\subsection{The Tree Layer}

The tree layer is concerned with a self-stabilizing algorithm for constructing a rooted spanning tree (see Figures 4 -a and 4-b)

\section{Interface and Behavior}

- (Exported Ports) Adjacent processes in the tree layer communicate via three ports: (1) pForest when two adjacent processes identify two different roots, (2) pNeighbor when two a parent and a child identify an inconsistency between them (i.e., existence of multiple roots, incorrect shortest distance to the root, or a root process that is not self-parent), and (3) $p P c$ when a parent process crashes. Port $p$ Cycle is used for cross-layer interactions described in Subsection 3.3.

- (Variables) Each tree component maintains the following variables: (1) an integer index to represent the unique index of the component, (2) an integer $f$ to keep the index of the parent process in the spanning tree, (3) an integer root that contains the index of the root process, and an integer $d$ whose value is the distance of the process to the root. The value of index is equal to that of the corresponding wave component and is specified statically. The value of $f$, however, is determined at runtime across the tree layer. Thus, the tree and wave components of a process need to communicate to maintain consistency. We address this issue in Subsection 3.3. Each component also maintains an array $N$, which contains the index of all adjacent processes. 


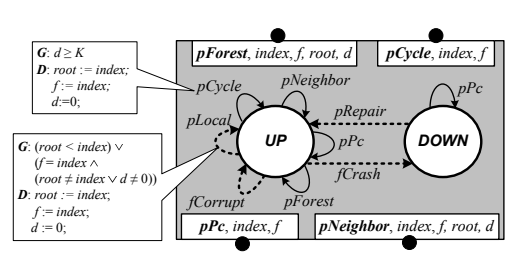

(a) Tree component

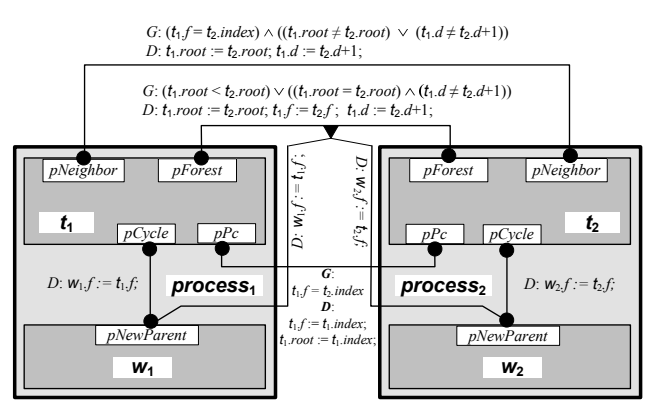

(b) The tree layer and cross-layer interactions

Fig. 4. The tree layer.

- (Automaton) Initially, all processes are alive and in the UP control state. Faults can alter the value of variables $f$, root, and $d$ arbitrarily through the internal port fCorrupt. Also, each process may crash and go to the control state Down through the internal port fCrash. A crashed process may get repaired and return to the UP control state through internal port pRepair. Thus, faults can potentially break a rooted tree into forests, create cycles, and cause (local or global) inconsistencies. A tree component participates in resolving the above issues when it is in control state UP. A local inconsistency is detected in a tree component through the internal port $p$ Local associated with a guard which indicates a discrepancy in the value of either root or $d$. A cycle can also be detected locally, if the distance of a process to the root is greater than the maximum number of processes $K$. A tree component fixes a local inconsistency and breaks a cycle by setting root $=f=$ index and $d=0$.

\section{Interactions}

Similar to the wave layer, interactions among tree components are specified in terms of a set of connectors between them according to a topology. Let $t$ be a tree component whose adjacent processes are $t_{1} . . t_{n}$. The interactions between tree components resolve the following issues to construct a rooted spanning tree. Recall that interactions between tree components construct Channel 1 of Figure 1-a:

- (Process crashes) The set $\left\{\left\langle(t . p P c)\left(t_{i} . p P c\right)\right\rangle \mid 1 \leq i \leq n\right\}$ of connectors are used to inform a process that its parent has crashed. As can be seen in Figure 4, this connector is enabled when one participating component is in UP and the other process is in DOWN control state. The guard of the connector enforces the parent-child relationship. Execution of this interaction invalidates the variables of the child process whose parent is crashed. 
- (Parental inconsistencies) A connector in the set $\left\{\left\langle(t . p N e i g h b o r)\left(t_{i} \cdot p N e i g h b o r\right)\right\rangle \mid 1 \leq i \leq n\right\}$ is enabled when a child and its parent either do not agree on the same root, or, the child is not located one step farther of its parent from the root. In either case, the child simply fixes the root index and its distance according to the parent through the data transfer mechanism of the connector (see the guard $G$ and transfer function $D$ of the connector in Figure 4-b).

- (Rooted forests) A connector in the set $\left\{\left\langle(\right.\right.$ t.pForest $)\left(t_{i} . p\right.$ Forest $\left.)\right\rangle \mid 1 \leq$ $i \leq n\}$ is enabled when multiple roots are detected by a tree component. This situation occurs when there exists an adjacent process whose root has a higher index or the process offers a shorter distance to the root. In this case, the process updates its root, $f$, and $d$ variables via the data transfer mechanism (see the guard $G$ and function $D$ of the connector in Figure 4-b).

Finally, we define the set of legitimate states of the tree layer, where a rooted tree that spans over all alive processes exists, as follows:

$$
\begin{aligned}
& \mathcal{S}_{t} \equiv(k=\max \{t \text {.index } \mid t . U P\}) \wedge \\
& \left(\forall t _ { 1 } | t _ { 1 } . \text { UP:: } \left(t_{1} \text {. index }=k \Rightarrow\right.\right. \\
& \left.\left(t_{1} \text {. index }=t_{1} \text {.root } \wedge t_{1} \text {.index }=t_{1} . f \wedge t_{1} . d=0\right)\right) \wedge \\
& \left(t_{1} \text {.index } \neq k \Rightarrow\right. \\
& \left(\exists t_{2} \in t_{1} . N::\left(t_{1} \cdot f=t_{2} . \text { index } \wedge t_{1} . d=t_{2} . d+1 \wedge\right.\right. \\
& \left.\left.\left.\left.\forall t_{3} \in t_{1} . N:: t_{2} . d \leq t_{3} . d\right)\right)\right)\right) \text {. }
\end{aligned}
$$

\subsection{Building Distributed Reset}

Given the tree layer and wave layer components, one can easily compose them and incrementally build a distributed reset system. To this end, we add cross layer interactions as follows. When a cycle or multiple forests are detected in the tree layer, a tree component may choose a new parent from its neighbors. In this case, the wave component of the same process has to update its parent as well, so the subsequent resets complete maturely (see Channel 2 in Figure 1-a). Thus, we augment each wave component with a pNewParent port, which synchronizes with $p$ Cycle or an exported port by the pForest connectors to update its parent (see Figure 4-b).

\section{Model Checking Distributed Reset}

In this section, we describe our technique to verify the correctness of distributed reset using classic model-checking. For a finite instantiation of the algorithm by a grid topology, we start by constructing a finite representation of its overall behavior as a flat labeled transition systems (LTS) using BIP state-space explorer [4]. States correspond to configurations reached by the algorithm, and transitions taken to move from one configuration to another are labeled by the interactions introduced in Section 3. On the LTS model, we have evaluated a set 
of temporal logic formulas encoding the key properties of distributed reset, using the Evaluator tool of CADP [14,18].

We express the properties using a generic characterization of interactions (i.e., labels). We note that given the set of legitimate states, such labeling can be easily automated in the context of verification of self-stabilizing algorithms:

- We add a self-loop labeled steady to each legitimate state. For the wave layer (respectively, tree layer), all these self-loops participate in a global rendezvous interaction whose guard satisfies expression $\mathcal{S}_{w}$ (respectively, $\mathcal{S}_{t}$ ) introduced in Section 3.

- We label each internal fault transition introduced in Section 3 by fault. This labeling makes the occurrence of a fault an observable event.

- We label the remaining interactions by prog. This includes recovery as well as interactions that participate in constructing a spanning tree at the tree layer and interactions that contribute in achieving a global reset at the wave layer.

We provide the exact definition of properties in regular alternation-free $\mu$ calculus which is the temporal logic formalism handled by the Evaluator tool. This logic is an extension of the alternation-free $\mu$-calculus [16] with action formulas as in ACTL [19] and regular expressions over action sequences as in PDL [13]. The full syntax and semantics can be found in [18]. We consider the following properties that any self-stabilizing system must satisfy:

- (closure) legitimate states are preserved by taking non-fault actions (only faults may reach an illegitimate state from a legitimate state):

$\phi_{1}:\left[\right.$ any $\left.{ }^{*}\right](\langle\text { steady }\rangle \mathbf{T} \Rightarrow[\text { prog }]\langle\text { steady }\rangle \mathbf{T})^{3}$

- (deadlock-freedom) from any reachable state, there exists an outgoing program transition:

$\phi_{2}:\left[a n y^{*}\right]\langle\operatorname{prog}\rangle \mathbf{T}$

- (reachability) starting from any state, a legitimate state can be reached by taking only program actions (there always exist a path from any state to a legitimate state): $\phi_{3}:\left[\right.$ any $\left.^{*}\right]\left\langle\right.$ prog $\left.^{*}\right\rangle\langle$ steady $\rangle \mathbf{T}$

- (convergence) starting from any state, a legitimate state is eventually reached by taking only program actions (the algorithm never reaches a cycle outside legitimate states):

$\phi_{4}:\left[\right.$ any $\left.^{*}\right] \neg \nu X .(\neg\langle$ steady $\rangle \mathbf{T} \wedge\langle\operatorname{prog}\rangle X)$

In order to reduce the complexity of verification of distributed reset, we utilize a compositional approach. Specifically, we infer the correctness of the composite

\footnotetext{
${ }^{3}$ We recall that $q \models\langle a\rangle \varphi$ iff $\exists q \stackrel{a}{\rightarrow} q^{\prime}: q^{\prime} \models \varphi$, where $q$ and $q^{\prime}$ are two states, $\stackrel{a}{\rightarrow}$ is a transition labeled by $a$, and $\varphi$ is a formula. Also, $q \models[a] \varphi$ iff $\forall q \stackrel{a}{\rightarrow} q^{\prime}: q^{\prime} \models \varphi$. The label any denotes any transition label, i.e., \{steady, prog, fault $\}, \mathbf{T}$ denotes logical true, and $*$ denotes a sequence. Finally, $\nu$ and $\mu$ respectively denote the largest and smallest fixpoints in the $\mu$-calculus.
} 


\begin{tabular}{|c|c||c|c|c||c|c|c|c|}
\hline \hline & $n$ & states & transitions & generation time & $\phi_{1}$ & $\phi_{2}$ & $\phi_{3}$ & $\phi_{4}$ \\
\hline \multirow{4}{*}{ tree } & 4 & 56 & 649 & $<1$ & $<1$ & $<1$ & $<1$ & $<1$ \\
\cline { 2 - 8 } & 6 & 7022 & 81390 & 29 & 1 & 1 & 2 & 3 \\
\cline { 2 - 8 } & 9 & 2456936 & 59409357 & 4000 & 10 & 23 & 19 & 145 \\
\hline \hline \multirow{3}{*}{ wave } & 4 & 996 & 5840 & $<1$ & $<1$ & $<1$ & $<1$ & $<1$ \\
\cline { 2 - 8 } & 6 & 27590 & 189523 & 36 & 2 & 2 & 3 & 5 \\
\cline { 2 - 9 } & 9 & 1539001 & 7077649 & 2500 & 5 & 7 & 6 & 93 \\
\hline \hline
\end{tabular}

Table 1. Verifying distributed reset using classic model checking.

distributed reset algorithm by verifying the correctness of the tree layer and wave layer individually. However, such compositional verification needs demonstration of interference-freedom between components. Let $C_{1}$ and $C_{2}$ be two components. We say that $C_{1}$ and $C_{2}$ do not interfere with each other if whenever $C_{1}$ satisfies some property $\varphi$ and $C_{2}$ satisfies some property $\varphi^{\prime}$, then their "composition" (e.g., using BIP interactions) satisfies $\varphi \wedge \varphi^{\prime}$.

Theorem 1. The composition of the tree layer and wave layer in the distributed reset algorithm is interference-free for properties $\phi_{1} \ldots \phi_{4}$.

Proof. Notice that the only interaction between the tree layer and wave layer occurs when a change of parent is decided by the tree layer. This interaction only involves a unilateral change of parent at the wave layer by the tree layer. Thus, the wave layer does not interfere with the tree layer in any way. Moreover, when the wave layer is silent, a change of parent does not change the state of the wave layer. Thus, the only possible pitfall of the aforementioned interaction is where an ongoing reset at the wave layer coincides with a change of parent at the tree layer. Since there exist only a finite number of actions at the tree layer to construct a spanning tree, the wave layer eventually complete its execution on the current spanning tree as well. The only consequence of a change of parent is that the ongoing reset completes immaturely, which is a known and permitted phenomenon in the original algorithm as well.

The immediate consequence of Theorem 1 is that we can verify the correctness of the layers of distributed reset independently. In order to generate LTS models of manageable size for a reasonably large number of processes in the algorithm we manually applied the following model checking heuristics on the BIP model:

- We apply abstraction by reducing the domain of values of each variable to the minimal possible set. For instance, when a fault alters the value of the root variable in a process, the exact new value does not matter and, hence, the corresponding illegitimate state can be encoded by a single corrupted value for the root variable. 


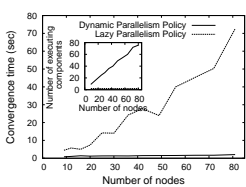

(a) Degree of parallelism in tree layer

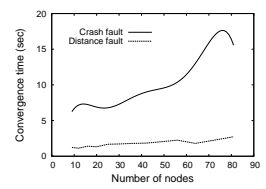

$\begin{array}{lr}\text { (b) } & \text { Crash } \\ \text { fault } & \text { vs. } \\ \text { variable } & \text { cor- } \\ \text { ruption } & \text { in } \\ \text { tree layer } & \end{array}$

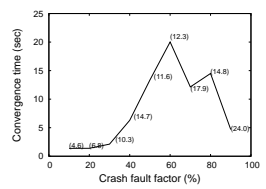

(c) Effect of fault factor in tree layer.

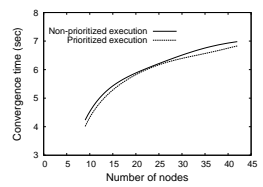

(d) Effect of priorities tree layer and wave layer.

Fig. 5. Performance analysis.

- We perform a live analysis [9] in every component and based on it, we reinitialize each variable as soon as it becomes dead on a computation path.

- Finally, we simplify the sequence of occurrence of faults by allowing multiple types of faults occurring at the same time.

Table 1 summarizes the results about the size of the models in terms of number of processes in the grid. The LTS generation time as well as the time needed to verify the properties considered are all in seconds. All verification tasks are run on a PC with a $3.2 \mathrm{GHz}$ Intel Xeon processor and 4GB RAM.

\section{Performance Evaluation}

The BIP toolset provides us with means for generating $\mathrm{C}++$ multi-threaded [3] as well as distributed [7] code from high-level BIP models. This feature enables us to evaluate the performance of distributed algorithms described by high-level models. This allows in particular, to evaluate the impact of changes to the highlevel model without getting involved with its actual $\mathrm{C}++$ implementation. We emphasize that the logical properties and dynamics of the $\mathrm{C}++$ model conform with the high-level model and an actual $\mathrm{C}++$ implementation. Below, we present the result of some of our experiments and lessons learned in evaluating the performance of distributed reset. We use the multi-threaded $\mathrm{C}++$ code in order to conduct guided simulations. All experiments in this section are run on a PC with a Pentium IV 3GHz processor and 1GB memory under Debian Linux. All plots on each graph is the average value of 10 runs for the corresponding experiment. The reason for this number of experiments is due to the fact that our models do not exhibit a high level non-determinism. In fact, we observed that the result of experiments do not fluctuate significantly.

Degree of Parallelism. The BIP Engine uses different parallelism policies to execute distributed models. In a lazy policy, the Engine executes only one 
interaction at a time. In other words, it waits for all atomic components to complete their internal computation before initiating a new interaction. Conversely, in a dynamic policy, the Engine allows multiple interactions to be executed in parallel as long as the overall execution conforms with the sequential semantics (i.e., their executions are observationally equivalent). Figure 5-a compares the convergence time of the tree layer under these policies in the absence of faults. As can be seen, the graph shows that under the dynamic policy convergence is much faster, as the Engine allows multiple tree components to work simultaneously. This makes performance evaluation of distributed algorithms very close to reality.

Severity of Faults. Figure 5-b compares the effect of $d$-variable corruption and crash faults on convergence time of the tree layer. The graphs clearly show that crash faults' damage to the spanning tree is more severe than the case where a process has wrong coordinates of the root. This result is expected, as crashing a node requires reconstructing the spanning tree, which can be costly. For instance, if a crashed process is the root, the entire spanning tree has to be reconstructed. On the other hand, a $d$-variable corruption can be fixed by a single interaction with one of the adjacent processes.

Figure 5-c shows the behavior of the tree layer in the presence of crash faults, where the probability of occurrence of such faults decreases by a fault factor ff, where $f f<1$. That is, if the current probability of a crash is $p$ for a process, after the process is repaired, the probability of the subsequent crash for this process is $f f * p$. As can be seen, the convergence time increases as the fault factor grows to $60 \%$. However, when the fault factor grows beyond $60 \%$, the tree layer converges faster. This is because there are so many crashed processes that are not repaired and, hence, not participating in forming a spanning tree. Thus, a high fault factor reduces the size of actual distributed system (the average number of process crashes for some plots are available in Figure 5-c).

Effect of specifying priorities. Figure 5-d shows the effect of granting priority to execution of tree layer over the wave layer. The idea is when the spanning is broken, the algorithm should focus on reconstructing a new tree rather than letting the wave layer work. In fact, simultaneous operation of both layers may result in completing immature resets. In BIP, one can easily specify priorities among interactions. In particular, we specify a local priority for the tree layer interactions of adjacent processes and Figure 5 -d shows slightly faster convergence for the prioritized tree layer.

\section{Conclusion}

The paper illustrates the application of a methodology consistently integrating high-level modeling and verification of functional properties with performance analysis of a distributed implementation in the BIP framework. BIP allows a natural high-level description of the coordination between atomic components 
by using structured connectors and multiparty interactions. Consistency is ensured by results guaranteeing preservation of properties of the initial high-level model by its implementation. We demonstrated how one can build-up the selfstabilizing distributed reset algorithm [2] by developing a set of independent atomic components and then wiring them by using connectors by considering functional and recovery tasks independently. We also identified a set of safety and liveness properties that any self-stabilizing algorithm has to satisfy: closure, deadlock-freedom, and finite reachability of the set of legitimate states starting from any arbitrary state. We successfully verified these properties for each layer of distributed reset for a grid topology. For performance evaluation, we used an automatically generated multi-threaded code observationally equivalent to the high-level BIP model. The obtained benchmarks show the effect of scheduling policies and of different types of faults on convergence times and the degree of parallelism. Here again incremental description by adding or removing architectural features has been very useful for modifying the model.

As illustrated in the paper, our approach advantageously combines an expressive and rigorous high-level component-based formalism and its associated distributed implementation. This is extremely beneficial for design and implementation of complex concurrency control algorithms. In this context, we are currently working on a generic component-based framework for modeling and analyzing transactional memory $[15,21]$ algorithms using BIP. We are also working on a wide range of transformations from high-level BIP models into low-level actual implementations such as the Message Passing Interface (MPI), multi-core, and fully distributed platforms. Another interesting research direction is to automate the procedure presented in this paper by transforming algorithms in (shared memory) guarded commands into BIP models.

\section{References}

1. M. Alexander and W. Gardner. Process Algebra for Parallel and Distributed Processing. Chapman \& Hall/CRC, 2008.

2. A. Arora and M. Gouda. Distributed reset. IEEE Transactions on Computers, 43:316-331, 1994.

3. A. Basu, P. Bidinger, M. Bozga, and J. Sifakis. Distributed semantics and implementation for systems with interaction and priority. In Formal Techniques for Networked and Distributed Systems (FORTE), pages 116-133, 2008.

4. A. Basu, M. Bozga, and J. Sifakis. Modeling heterogeneous real-time components in BIP. In Software Engineering and Formal Methods (SEFM), pages 3-12, 2006.

5. S. Bliudze and J. Sifakis. Causal semantics for the algebra of connectors. In Formal Methods for Components and Objects (FMCO), pages 179-199, 2007.

6. S. Bliudze and J. Sifakis. A notion of glue expressiveness for component-based systems. In Concurrency Theory (CONCUR), pages 508-522, 2008.

7. B. Bonakdarpour, M. Bozga, M. Jaber, J. Quilbeuf, and J. Sifakis. Automated conflict-free distributed implementation of component-based models. In IEEE Symposium on Industrial Embedded Systems (SIES), 2010. To appear.

8. B. Bonakdarpour, M. Bozga, M. Jaber, J. Quilbeuf, and J. Sifakis. From high-level component-based models to distributed implementations. In ACM International Conference on Embedded Software (EMSOFT), 2010. To appear. 
9. M. Bozga, J.-C. Fernandez, and L. Ghirvu. State-space reduction based on live variable analysis. Journal of Science of Computer Programming, 47(2-3):203-220, 2003.

10. K. M. Chandy and J. Misra. Parallel program design: a foundation. AddisonWesley Longman Publishing Co., Inc., Boston, MA, USA, 1988.

11. E. W. Dijkstra. Self-stabilizing systems in spite of distributed control. Communications of the ACM, 17(11):643-644, 1974.

12. E. W. Dijkstra. A belated proof of self-stabilization. Distributed Computing, 1(1):5$6,1986$.

13. M. J. Fischer and R. E. Ladner. Propositional Dynamic Logic of Regular Programs. Journal of Computer and System Sciences, 18:194-211, 1979.

14. H. Garavel, F. Lang, R. Mateescu, and W. Serve. CADP 2006: A Toolbox for the Construction and Analysis of Distributed Processes. In W. Damm and H. Hermanns, editors, Computer Aided Verification (CAV), pages 158-163, 2007.

15. M. Herlihy and J. E. B. Moss. Transactional memory: Architectural support for lock-free data structures. In ISCA, pages 289-300, 1993.

16. D. Kozen. Results on the propositional $\mu$-calculus. Theoretical Computer Science, 27:333-354, 1983.

17. N. Lynch. Distributed Algorithms. Morgan Kaufmann Publishers, San Mateo, CA, 1996.

18. R. Mateescu and M. Sighireanu. Efficient On-the-Fly Model-Checking for Regular Alternation-Free Mu-Calculus. Science of Computer Programming, 46(3):255-281, 2003.

19. R. D. Nicola and F. Vaandrager. Action versus State Based Logics for Transition Systems. In Semantics of Systems of Concurrent Processes (La Roche Posay, France), pages 407-419, 1990.

20. T. N. S. Bensalem, M. Bozga and J. Sifakis. D-finder: A tool for compositional deadlock detection and verification. In Computer Aided Verification (CAV), pages 614-619, 2009.

21. N. Shavit and D. Touitou. Software transactional memory. Distributed Computing, 10(2):99-116, 1997.

22. J. Sifakis. A framework for component-based construction extended abstract. In Software Engineering and Formal Methods (SEFM), pages 293-300, 2005.

23. J. A. Tauber, N. A. Lynch, and M. J. Tsai. Compiling IOA without global synchronization. In Symposium on Network Computing and Applications (NCA), pages 121-130, 2004. 